\title{
Triagem de hipotireoidismo em crianças - Visão dos médicos da atenção primária à saúde de um município de médio porte
}

\author{
Hypothyroidism screening in children: Views of primary health care physicians from a
} medium-sized city

\section{Detección de hipotiroidismo en niños - Visión de los médicos de atención primaria a la salud de un municipio de tamaño medio}

Nathalia Bordeira Chagas. Departamento de Medicina, Universidade Federal de São Carlos (UFSCar). São Carlos, SP, Brasil. na.bordeira@uol.com.br

Leonardo Antonelli. Departamento de Medicina, Universidade Federal de São Carlos (UFSCar). São Carlos, SP, Brasil. leonardo-antoneli@hotmail.com

Vanessa Furino. Programa de Pós-Graduação em Enfermagem (PPG-Enf), Universidade Federal de São Carlos (UFSCar). São Carlos, SP, Brasil. vafurino@ hotmail

Debora Gusmão Melo. Departamento de Medicina, Universidade Federal de São Carlos (UFSCar). São Carlos, SP, Brasil. debora.gusmao@gmail.com

Carla Maria Ramos Germano. Departamento de Medicina, Universidade Federal de São Carlos (UFSCar). São Carlos, SP, Brasil.cgermano@ufscar.br (Autora correspondente)

\section{Resumo}

Objetivo: Avaliar quais parâmetros os médicos da atenção primária à saúde do município de São Carlos, SP, Brasil, adotam para realizar triagem de função tireoidiana em crianças e adolescentes, quais exames complementares são solicitados e qual a conduta diante de resultados laboratoriais alterados. Métodos: Estudo transversal descritivo, no qual participaram 26 profissionais, correspondendo a $96,3 \%$ de todos os médicos da atenção primária da cidade que atuam no cuidado de crianças e adolescentes. Os participantes responderam por escrito quais as três situações mais frequentes na prática clínica em que indicavam triagem de hipotireoidismo na população pediátrica, quais os exames laboratoriais solicitados nesta avaliação e a qual conduta frente a exames com resultados alterados. Resultados: As situações clínicas mais frequentes apontadas pelos participantes como indicações de avaliação tireoidiana foram: obesidade, com 23 respostas (88,5\%); baixa estatura, com 16 (61,5\%); e síndrome de Down, com $8(30,8 \%)$. Nove médicos $(34,6 \%)$ responderam realizar o rastreio pela dosagem de TSH e T4I e outros $9(34,6 \%)$, pela dosagem de TSH isolado. A conduta mais frequente diante de um resultado alterado foi o encaminhamento ao endocrinologista pediátrico, com 14 respostas (53,8\%). Conclusão: O cenário encontrado neste estudo mostra que exames de função tireoidiana são solicitados rotineiramente por médicos da atenção primária no acompanhamento de crianças obesas. Como a obesidade infantil tem uma alta prevalência, isso pode determinar aumento importante dos custos do serviço de saúde e sobrecarga nos serviços de atenção especializada.
Palavras-chave:

Hipotireoidismo

Criança

Triagem

Atenção Primária à Saúde
Como citar: Chagas NB, Antonelli L, Furino V, Melo DG, Germano CMR. Triagem de hipotireoidismo em crianças - Visão dos médicos da atenção primária à saúde de um município de médio porte. Rev Bras Med Fam Comunidade. 2016;11(38):1-10. http://dx.doi.org/10.5712/rbmfc11(38)1088
Fonte de financiamento: CAPES.

Parecer CEP: 580.506 (UFSCar), aprovado em 08/04/2014.

Conflito de interesses: declaram não haver. Procedência e revisão por pares: revisado por pares. Recebido em: 09/03/2015. Aprovado em: 26/01/2016. 


\section{Abstract}

Objective: To determine which parameters are used by primary care physicians of São Carlos city, SP, Brazil, to screen thyroid function in children and adolescents, which tests are required for the assessment, and what options are provided to patients with abnormal results. Methods: This was a descriptive study in which 26 professionals participated, corresponding to $96.3 \%$ of all primary care physicians who provide medical care to children and adolescents. The participants provided written answers for questions regarding the 3 most common situations in clinical practice that warranted a hypothyroidism screening test in the pediatric population; the laboratory tests required for the assessment, and the options provided to patients with abnormal results. Results: The most common clinical situations identified by the physicians as indications for evaluation of thyroid function were obesity ( 23 answers; $88.5 \%$ ), short stature (16 answers; $61.5 \%$ ), and Down syndrome (8 answers; $30.8 \%$ ). Nine physicians (34.6\%) screened thyroid function by using the TSH and FT4 tests, whereas 9 other physicians (34.6\%) used only the TSH test. In case of abnormal results, most physicians referred the patient to a pediatric endocrinologist (14 answers; $53.8 \%$ ). Conclusion: Thus, the findings of this study indicate that thyroid function tests are routinely ordered by primary care physicians for the follow-up of obese children. As childhood obesity is highly prevalent, it may lead to a significant increase in health service costs and overload secondary care services.

\section{Resumen}

Objetivo: Evaluar los parámetros que los médicos de atención primaria de salud en la ciudad de São Carlos, SP, Brasil, adoptan para realizar exámenes de función de la tiroides en niños y adolescentes, los exámenes complementarios necesarios y cual conducta ante los resultados anormales de laboratorio. Métodos: Estudio descriptivo, que asistieron 26 profesionales, representando $96,3 \%$ de todos los médicos de atención primaria de la ciudad que trabajan con niños y adolescentes. Ellos respondieron cuales son las tres situaciones más comunes en la práctica clínica que indican cribado de hipotiroidismo en la población pediátrica, que exámenes adicionales solicitados en esta evaluación y cual conducta delante los exámenes laboratoriales con resultados anormales. Resultados: Las situaciones clínicas más frecuentes identificadas por los participantes como indicadoras de evaluación de la tiroides son: la obesidad con 23 respuestas $(88,5 \%)$, baja estatura, con $16(61,5 \%)$ y el síndrome de Down con $8(30,8 \%) .9(34,6 \%)$ médicos dijeron realizar rastreo por medio de la TSH y T4 libre y otros $9(34,6 \%)$, a través de la dosis de TSH aislado. La conducta más mencionada ante un resultado anormal fué un encaminamiento al endocrinologista pediatra, con 14 respuestas (53,8\%). Conclusión: El escenario en este estudio muestra que los exámenes de función de la tiroide son solicitados rutinariamente por los médicos de atención primaria en el acompañamiento de niños obesos. Como la obesidad infantil tiene una alta prevalencia, esto puede causar incremento en los costos de los servicios de salud y en las despensas generales en los servicios de atención especializada.

\section{Keywords:}

Hypothyroidism

Child

Screening

Primary Health Care

Palabras clave:

Hipotiroidismo

Niño

Triaje

Atención Primaria de Salud

\section{Introdução}

O hipotireoidismo, patologia causada pela deficiência da produção ou da ação dos hormônios tireoidianos, tem prevalência comum na infância. ${ }^{1}$ A prevalência dessa desordem adquirida após o nascimento tem sido reportada entre 1,7\% e 9,5\% em crianças e adolescentes, sendo mais frequente em meninas do que em meninos. ${ }^{2}$

As indicações de investigação diagnóstica na população pediátrica compreendem a presença de um conjunto de sinais e sintomas compatíveis com o quadro clínico de hipotireoidismo franco, ${ }^{3}$ representados principalmente por prejuízo no crescimento e atraso na maturação óssea, atraso no desenvolvimento neuropsicomotor e/ou atraso escolar, bradicardia, palidez, pele e cabelos secos, obstipação intestinal, intolerância ao frio, mixedema, puberdade atrasada e irregularidade menstrual. ${ }^{1}$ Algumas condições clínicas também podem estar associadas com disfunção tireoidiana como as síndromes de Down e de Turner, diabetes melito tipo 1, dislipidemia e beta talassemia maior. ${ }^{4}$ Nesses casos, o rastreio periódico para hipotireoidismo está indicado, mesmo em indivíduos assintomáticos.

O público leigo e alguns profissionais de saúde consideram que o hipotireoidismo está associado com obesidade, atribuindo à hipofunção tireoidiana a causa do peso excessivo. Não existem, porém, dados científicos que fundamentem essa hipótese. ${ }^{3,5} \mathrm{Na}$ prática clínica, é descrito um número crescente de testes de função tireoidiana solicitados na faixa pediátrica, sobretudo em situações em que o hipotireoidismo 
não é frequente. ${ }^{3}$ Diante deste cenário, o presente estudo pretendeu avaliar a concepção de médicos da atenção primária à saúde, de um município de médio porte do Brasil, a respeito dos parâmetros referenciais adotados para a triagem de hipotireoidismo em crianças e adolescentes, e confrontar os achados com as indicações preconizadas atualmente na literatura científica.

\section{Métodos}

Trata-se de um estudo transversal de natureza descritiva e exploratória, previamente aprovado pelo Comitê de Ética em Pesquisa com Seres Humanos da UFSCar (parecer no 580.506) e conduzido durante o ano de 2014. Foram convidados a participar da pesquisa todos os médicos da atenção primária à saúde que atuavam no cuidado de crianças e adolescentes na cidade de São Carlos, SP, Brasil (27 profissionais).

Localizada no centro geográfico do Estado de São Paulo, São Carlos tem cerca de 220.000 habitantes, aproximadamente 2.700 nascimentos/ano ${ }^{6}$ e em 2010 apresentou índice de desenvolvimento humano de 0,805, sendo considerado o 28ํㅜ município mais desenvolvido do país. ${ }^{7}$ No município, a Atenção Primária à Saúde está baseada em 12 Unidades Básicas de Saúde (UBS) e 17 Unidades de Saúde da Família.

Todos os médicos que atendem crianças e adolescentes, no contexto da atenção primária em saúde do município, foram convidados para participar do estudo. Dos 27 médicos convidados a participar, apenas um se recusou, resultando em uma amostra representativa de $96,3 \%$ da população alvo. A participação foi condicionada à assinatura de Termo de Consentimento Livre e Esclarecido.

Os 26 profissionais que concordaram em participar do estudo responderam a três questões elaboradas como instrumento de coleta de dados, aplicadas por um estudante de graduação em Medicina. O estudante abordou individualmente cada participante, apresentou o TCLE, resolveu dúvidas sobre a pesquisa, entregou uma folha com as questões impressas e deixou que os participantes respondessem por escrito às questões, sem sua intervenção. As folhas com as respostas foram recolhidas, após o término de seu preenchimento, para análise posterior.

Os participantes responderam às seguintes questões:

1. Quais as três situações mais frequentes na prática clínica em que você indica rastreio de função tireoidiana em crianças e adolescentes, sem sintomas de hipotireoidismo franco?

2. Que exames laboratoriais solicita para realizar esta triagem?

3. Qual sua conduta frente a um resultado laboratorial alterado?

Os dados coletados foram compilados, categorizados e apresentados como valores absolutos e porcentagem.

\section{Resultados}

Entre os 26 participantes, 13 (50\%) eram do sexo feminino e 13 (50\%), do sexo masculino. A distribuição etária compreendeu 7 (26,9\%) com idade menor ou igual a 40 anos, e 19 (73,1\%) com idade maior que 40 anos. Dezesseis dos médicos entrevistados (61,5\%) se formaram antes de 1995 , e 10 $(38,5 \%)$, após essa data. Com relação ao local de atuação, $15(57,7 \%)$ trabalhavam em UBS e $11(42,3 \%)$, em USF, e à especialidade médica, 15 (57,7\%) possuíam formação em pediatria; 6 (23,1\%), em saúde da família; 3 (11,5\%), em pediatria e saúde da família, e o restante $(7,7 \%)$ não possuía especialidade. 0 perfil da amostra pode ser visualizado no Quadro 1. 
Quadro 1. Perfil da amostra dos médicos entrevistados com relação à especialidade médica.

\begin{tabular}{|lc|}
\hline Especialidade médica & Número (\%) \\
\hline Pediatria & $15(57,7 \%)$ \\
Saúde da família & $6(23,1 \%)$ \\
Pediatria e Saúde da família & $3(11,5 \%)$ \\
Sem especialidade & $2(7,7 \%)$ \\
\hline
\end{tabular}

Da análise dos questionários, as situações clínicas apontadas pelos participantes como as mais frequentes indicações de avaliação tireoidiana em crianças sem sintomatologia de hipotireoidismo franco foram: obesidade, com 23 respostas (88,5\%); baixa estatura, com 16 (61,5\%); e síndrome de Down, com 8 (30,8\%). Outras situações clínicas também foram citadas, como mostrado na Figura 1.

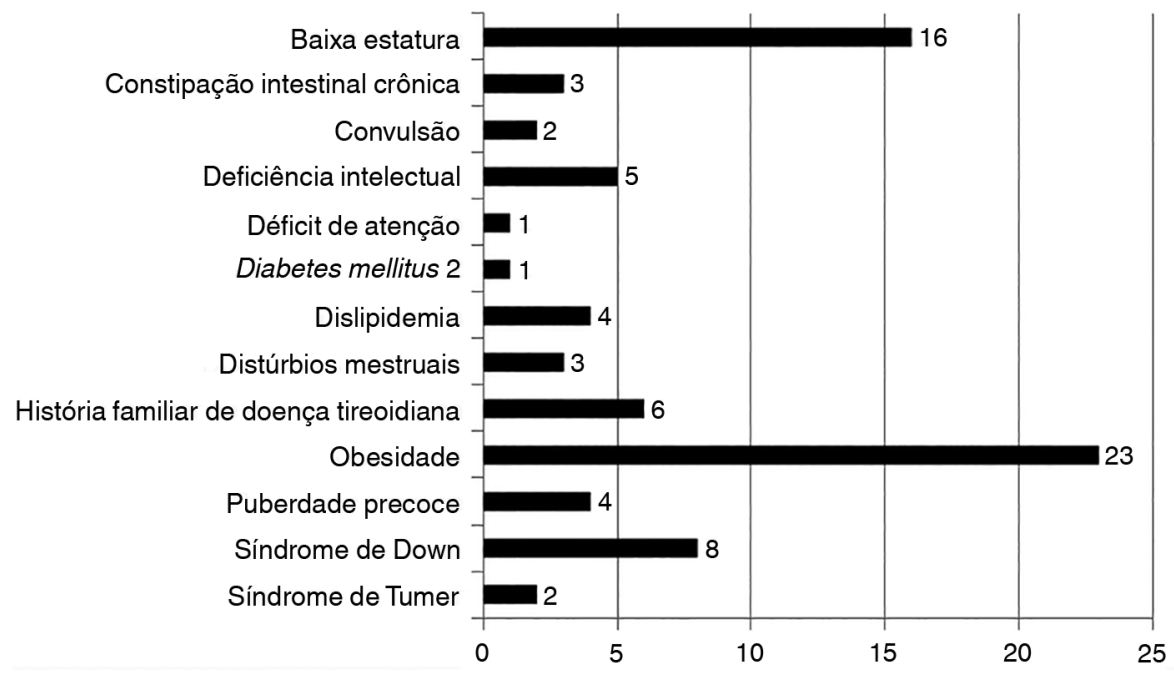

Figura 1. Distribuição em números das situações clínicas mais frequentes que os médicos entrevistados consideraram como indicação de rastreio de função tireoidiana em pacientes assintomáticos.

* Um profissional respondeu quatro indicações e outro respondeu apenas duas.

A respeito do exame solicitado para rastreio inicial, nove (34,6\%) médicos responderam dosar TSH e T4I; outros nove (34,6\%), somente TSH, e cinco (19,2\%) TSH, T4I e triiodotironina (T3). Houve também respostas que compreendiam a dosagem de TSH acrescida de T3 ou T4 total, como mostra a Figura 2.

Por fim, a conduta mais prevalente diante de um resultado de exame laboratorial alterado foi o encaminhamento ao endocrinologista pediatra, com 14 respostas (53,8\%). Outras condutas também apareceram entre as respostas, como mostrado na Figura 3.

\section{Discussão}

O hipotireoidismo subclínico (HS), definido como elevação isolada de TSH com níveis normais de T3 e T4, é considerado como fator de risco para evolução ao hipotireoidismo franco e para outras disfunções orgânicas, como alterações no metabolismo de carboidratos e de lipídeos e doenças cardiovasculares. ${ }^{1} \mathrm{~A}$ escassez de estudos controlados reportando o curso clínico natural do HS na população pediátrica, e suas consequências para a saúde futura desses indivíduos, ${ }^{3}$ justifica a importância de se realizar a triagem da função tireoidiana de acordo com as indicações preconizadas cientificamente, para que um diagnóstico e terapêutica adequados possam ser instituídos. 


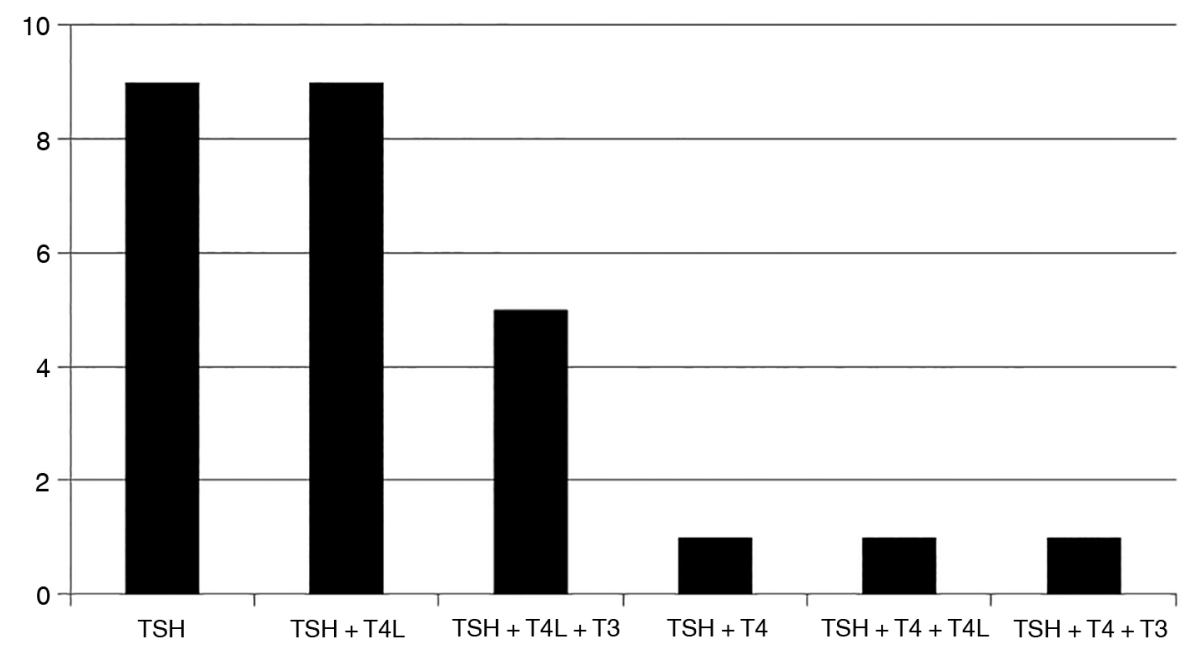

Figura 2. Distribuição, em números absolutos, dos exames solicitados pelos médicos entrevistados, para avaliação de função tireoidiana.

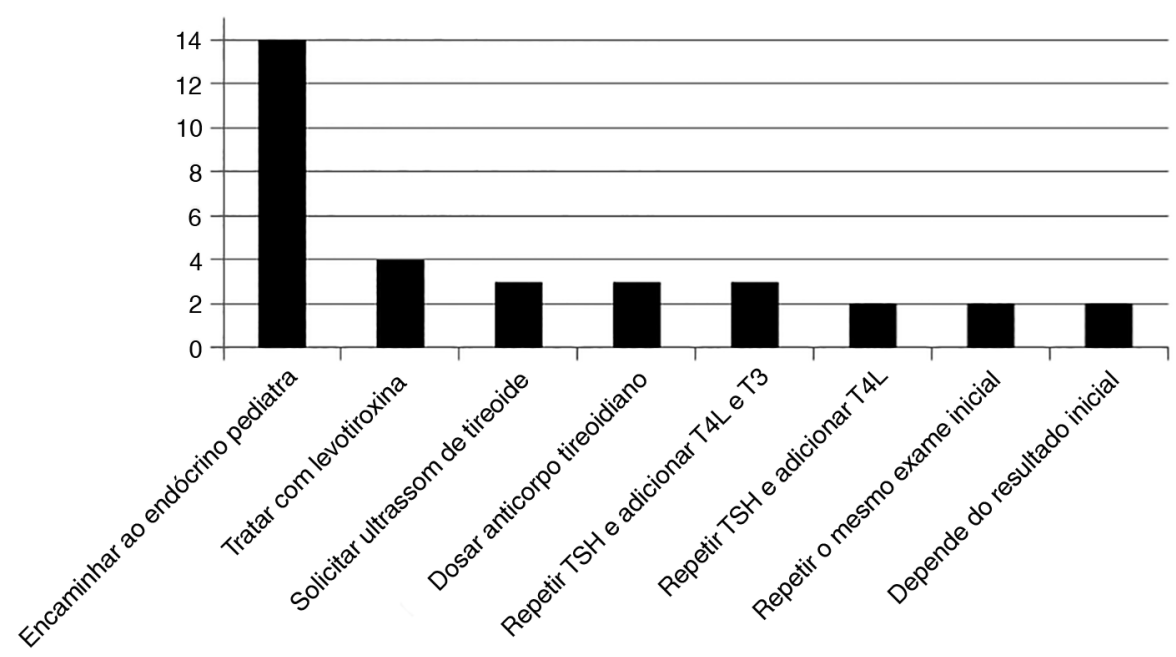

Figura 3. Distribuição em números absolutos da conduta dos profissionais médicos frente a um resultado alterado de função tireoidiana.

O presente estudo buscou identificar que parâmetros referenciais os profissionais médicos da atenção primária à saúde adotam para rastreio de hipotireoidismo em crianças e adolescentes. Obesidade foi encontrada como indicação clínica mais prevalente, seguida por baixa estatura e síndrome de Down.

Estudos recentes indicam que obesidade não é fator causal de hipotireoidismo e não se configura como uma indicação de triagem de disfunção tireoidiana, embora na prática seja frequentemente observada solicitação dessa triagem em crianças obesas como rotina. ${ }^{3,8}$ Assim como em nosso estudo, O' Brien et al. ${ }^{9}$ encontraram que a maioria dos médicos da atenção primária incluía a avaliação da função tireoidiana como rotina no cuidado de pacientes obesos e salientaram a ausência dessa indicação pela Academia Americana de Pediatria, devido à infrequente associação do hipotireoidismo como causa de obesidade isolada. Outros estudos mostraram que níveis séricos de TSH elevados associados com T4 livre normal foram encontrados em indivíduos obesos, sendo os valores de TSH diretamente correlacionados com os do IMC. ${ }^{10-15}$ 
Esse aumento de TSH, porém, se mostrou reversível com a perda de peso - o que suporta a teoria de que a hipertireotropinemia nessas crianças seja consequência da obesidade e não o oposto. 4,13,14,16 Reinehr et al. ${ }^{17}$ analisaram hormônios tireoidianos em um grupo de meninas adolescentes com obesidade e outro com anorexia nervosa (AN), antes e após a normalização do peso. Estes autores demonstraram que adolescentes obesas tinham aumento moderado de TSH e T3 livre, ao passo que meninas com AN tinham níveis de TSH e T3 livre ligeiramente diminuídos, quando comparadas com um grupo de meninas adolescentes saudáveis, de mesma idade e com peso normal. Essas alterações foram normalizadas nas adolescentes obesas com a perda de peso, e, nas meninas com AN, com o ganho de peso, sugerindo que os hormônios tireoidianos são reversivelmente relacionados com o peso corporal. ${ }^{10}$

A baixa estatura (BE), segunda situação clínica mais citada nessa pesquisa como indicação de rastreio, representa uma indicação de triagem legitimada pela literatura científica. Crianças e adolescentes com BE ou com velocidade de crescimento progressivamente diminuída e idade óssea atrasada, sem outros achados clínicos, podem apresentar HS. ${ }^{1}$ Os hormônios tireoidianos (HT) são fatores essenciais ao crescimento fisiológico, fazendo com que sua diminuição resulte em prejuízo do crescimento normal. ${ }^{1}$

A síndrome de Down, apontada pelos médicos participantes, também se enquadra como indicação para triagem de disfunção tireoidiana. O HS tem prevalência estimada entre $25,3 \%$ a $60 \%$ nessa população. ${ }^{18}$ Alguns estudos longitudinais demonstraram um risco dos indivíduos com síndrome de Down desenvolverem disfunção tireoidiana durante a vida próximo a 50\%. ${ }^{4}$ Assim, pacientes com síndrome de Down devem ser submetidos a rastreio periódico a cada dois anos com dosagem de hormônios tireoidianos para que, uma vez diagnosticados, possam ter o tratamento instituído precocemente. ${ }^{19,20}$

Outra síndrome que também tem associação com disfunção tireoidiana é a síndrome de Turner (ST). A prevalência de hipotireoidismo descrita em meninas com ST varia de $0 \%$ a $40 \% .{ }^{21}$ Devido ao risco aumentado de hipotireoidismo nessas pacientes, ${ }^{22}$ recentes guidelines internacionais trazem, entre outras recomendações, a dosagem de hormônios tireoidianos anualmente nesse grupo. ${ }^{23}$

Outras condições clínicas bem estabelecidas como indicação para triagem de hipotireoidismo são algumas doenças autoimunes. Pacientes com diabetes mellitus tipo 1 (DM 1) podem ter outras patologias endócrinas autoimunes associadas, sendo a disfunção tireoidiana uma das desordens mais frequente. ${ }^{21,24,25}$ A International Society for Pediatric and Adolescent Diabetes (ISPAD) recomenda que o rastreio da função tireoidiana seja realizado no momento do diagnóstico de DM 1 e, após, a cada dois anos em indivíduos assintomáticos, sem bócio e na ausência de anticorpos positivos. ${ }^{26} \mathrm{~A}$ Associação Americana de Diabetes (ADA) recomenda triagem da função tireoidiana após estabilização do quadro de diabetes, na presença de sintomas de disfunção tireoidiana, e a cada 1-2 anos se o resultado inicial for normal. ${ }^{27}$

Entre outras patologias autoimunes associadas à disfunção tireoidiana, estão a doença celíaca, o lúpus eritematoso sistêmico (LES) ${ }^{21,28}$ e a artrite idiopática juvenil (AIJ). ${ }^{29}$ Stagi et al.$^{30}$ sugerem monitorização cuidadosa para essa possível associação durante o curso da AIJ.

Os hormônios tireoidianos, dentre todas suas funções, desempenham um importante papel no metabolismo dos lipídeos, regulando as atividades dos receptores e das enzimas envolvidas no metabolismo das lipoproteínas. ${ }^{31}$ Assim, alterações no perfil lipídico podem ser decorrentes do hipotireoidismo. Catli et al. ${ }^{32}$ relatam que $1-11 \%$ de todos os pacientes com dislipidemia tem HS e Tanis 
et al. ${ }^{33}$, em sua metanálise, mostraram que o HS era duas a três vezes mais comum em pacientes com níveis séricos de colesterol total aumentados do que em pacientes com perfil lipídico normal. $A$ Associação Americana de Tireoide (ATA) recomenda que todos os pacientes com hipercolesterolemia sejam rastreados para disfunção tireoidiana. ${ }^{34}$

De maneira geral, a Sociedade Brasileira de Endocrinologia e Metabologia (SBEM) se posiciona contra a triagem universal para hipotireoidismo e recomenda o rastreio para o que denomina "grupos de alto risco", os quais compreendem mulheres grávidas e idosas, especialmente acima de 60 anos; indivíduos submetidos à radiação da glândula tireoidiana, cirurgia de tireoide prévia, disfunção anterior de tireoide, positividade do anticorpo ATPO, uso de alguns medicamentos como amiodarona ou lítio; portadores de DM 1, LES, síndrome de Sjögren, artrite reumatoide, SD, ST, vitiligo, insuficiência cardíaca, dislipidemia, hiperprolactinemia e anemia. Afirma, também, que a avaliação da função tireoidiana deve ser realizada em pacientes com sintomas clínicos de hipotireoidismo ou com história familiar de doença autoimune da tireoide, uma vez que há alta prevalência nesta última associação ${ }^{21}$. Não tem recomendações específicas de triagem para a população pediátrica.

As recomendações de seis organizações internacionais sobre a triagem da disfunção tireoidiana em indivíduos adultos assintomáticos estão resumidas abaixo (Quadro 2).

Quadro 2. Recomendação de 6 organizações internacionais sobre triagem de disfunção tireoidiana em indivíduos adultos assintomáticos (modificado de Garber et al., ${ }^{35}$ ).

\begin{tabular}{|ll|}
\hline Organização & Recomendações para triagem \\
\hline American Thyroid Association & Homens e mulheres acima de 35 anos, a cada 5 anos. \\
American Association of Clinical Endocrinologists & Pacientes mais velhos, especialmente mulheres. \\
American Academy of Family Physicians & Pacientes acima de 60 anos. \\
American College of Physicians & Mulheres acima de 50 anos com achados sugestivos de hipotireoidismo \\
U.S. Preventive Services Task Force & (percebidos durante a consulta clínica). \\
Royal College of Physicians of London & Evidências insuficientes contra ou a favor da triagem. \\
\hline
\end{tabular}

Os exames laboratoriais para investigação do hipotireoidismo em crianças e adolescentes compreendem a solicitação da dosagem sérica tanto de TSH quanto de T4I. ${ }^{20}$ Recomenda-se que, diante de valores laboratoriais alterados, nova função tireoidiana seja solicitada para descartar eventual erro de laboratório ou alteração transitória da função tireoidiana ${ }^{21,32}$ e, então, confirmar o diagnóstico de hipotireoidismo. Já em adultos, a SBEM recomenda que para triagem ou diante da suspeita de hipotireoidismo deve-se dosar inicialmente o TSH isolado e, se alterado e confirmado após 2-3 meses, o T4 livre deve, então, também ser dosado. ${ }^{21}$ Neste estudo prevaleceu a solicitação de TSH com T4I e de TSH isolado em iguais proporções, o que mostra que alguns profissionais procedem a investigação de disfunção tireoidiana na população pediátrica como recomendado para adultos.

A conduta frente a resultado de exame alterado que predominou neste estudo foi o encaminhamento imediato ao especialista, sem confirmação laboratorial. A dosagem de outros hormônios tireoidianos (T4 total e T3), assim como a solicitação de anticorpos tireoidianos e de ultrassonografia (USG) de tireoide também foram condutas adotadas pelos profissionais após um primeiro exame alterado. 
A respeito dos hormônios dosados na investigação diagnóstica, Kaplowitz afirma que os médicos da atenção primária devem ser orientados a solicitar apenas TSH e T4I, e que outros hormônios, como T3 e sua fração livre, só adicionam custo à triagem e frequentemente têm valores anormais em indivíduos sem patologia tireoidiana. ${ }^{3}$

A SBEM sugere que após confirmação laboratorial de hipotireoidismo, isto é, após exames iniciais alterados repetidos e confirmados, o passo seguinte é a investigação etiológica, que pode incluir a dosagem de anticorpos tireoidianos. ${ }^{21}$ Com relação ao uso de pesquisa dos anticorpos como teste de triagem, Kaplowitz afirma que há controvérsia a respeito de sua utilidade, uma vez que esses anticorpos podem ser positivos em crianças normais. ${ }^{3}$ Entretanto, um nível crescente do anticorpo anti-tireoperoxidase (TPO) é preditivo de disfunção tireoidiana iminente, ${ }^{4}$ podendo, assim, sua dosagem ser útil para orientar o acompanhamento e também a decisão terapêutica do HS. Quanto à USG de tireoide, a SBEM não recomenda sua realização de rotina. ${ }^{21}$

Com relação ao tratamento do HS, não há ainda um amplo consenso sobre quem deva ser tratado e qual nível de corte de TSH a ser utilizado em crianças. ${ }^{4} \mathrm{~A}$ recomendação atual é que o tratamento seja iniciado com TSH >10mIU/L, principalmente se esse valor for associado à positividade do anticorpo antiTPO. ${ }^{1,3,4}$ Vale lembrar que em cerca de $40 \%$ dos casos, o TSH pode normalizar espontaneamente, sem nenhuma intervenção, configurando um quadro transitório. ${ }^{1,3,4}$

Assim, diante de valores de TSH acima dos limites considerados normais e na ausência de fatores que justifiquem a terapêutica, recomenda-se a monitorização regular desses pacientes para que se possa detectar e tratar prontamente aqueles que evoluírem para um hipotireoidismo franco. Essa conduta expectante é possível, uma vez que estudos demonstram não haver prejuízo no desenvolvimento e crescimento dessas crianças com HS, ${ }^{3}$ e precisa fazer parte do conhecimento do médico da atenção primária que está envolvido diretamente no cuidado desses pacientes pediátricos.

\section{Conclusões}

O HS é uma patologia comum na população pediátrica e vem apresentando uma prevalência crescente, sobretudo pela realização de triagem da função tireoidiana em crianças e adolescentes sem uma indicação cientificamente fundamentada. ${ }^{32}$

Como a obesidade tem sido encontrada cada vez mais frequentemente na população pediátrica, a inapropriada prática de solicitação de exames de tireoide como procedimento de rotina para avaliação de pacientes obesos pode determinar um aumento importante dos custos do sistema público de saúde e sobrecarga dos serviços de atenção especializada. O mesmo se aplica para as demais situações que não se enquadram nas recomendações formais de triagem e que foram citadas pelos médicos entrevistados neste estudo. Além do aspecto financeiro, a demasiada solicitação de exames pode gerar ansiedade e preocupação desnecessárias aos pais e às crianças. Por outro lado, outras indicações importantes de triagem da função tireoidiana que não foram citadas pelos participantes do estudo, como DM1 e dislipidemia, devem fazer parte do conhecimento do médico da atenção primária, para que as patologias tireoidianas relacionadas a essas condições possam ser diagnosticadas precocemente e tratadas adequadamente. 
Uma vez realizado o rastreio com T4I e TSH, se o resultado for alterado, deve ser confirmado. Quanto à decisão de iniciar o tratamento do HS em crianças, Gawlik et al., ${ }^{36}$ em revisão recente, reafirmam que a qualidade dos estudos sobre o tema não é satisfatória, o que não permite chegar a conclusões definitivas sobre o assunto. Assim, novos estudos randomizados e em grande escala são necessários para determinar qual a melhor conduta para o paciente pediátrico com HS.

\section{Referências}

1. Setian NS. Hypothyroidism in children: diagnosis and treatment. J Pediatr (Rio J). 2007;83(5 Suppl):S209-16. DOI: http:// dx.doi.org/10.2223/JPED.1716

2. Biondi B, Palmieri EA, Lombardi G, Fazio S. Subclinical hypothyroidism and cardiac function. Thyroid. 2002;12(6):505-10. DOI: http://dx.doi.org/10.1089/105072502760143890

3. Kaplowitz PB. Subclinical hypothyroidism in children: normal variation or sign of a failing thyroid gland? Int $\mathrm{J}$ Pediatr Endocrinol. 2010;2010:281453. DOI: http://dx.doi.org/10.1155/2010/281453

4. O'Grady MJ, Cody D. Subclinical hypothyroidism in childhood. Arch Dis Child. 2011;96(3):280-4. DOI: http://dx.doi. org/10.1136/adc.2009.181800

5. Rapa A, Monzani A, Moia S, Vivenza D, Bellone S, Petri A, et al. Subclinical hypothyroidism in children and adolescents: a wide range of clinical, biochemical, and genetic factors involved. J Clin Endocrinol Metab. 2009;94(7):2414-20. DOI: http:// dx.doi.org/10.1210/jc.2009-0375

6. Brasil - IBGE [homepage on the Internet]. Censo Demográfico 2010 [accessed Mar 2 2014]. Available from: http://www. cidades.ibge.gov.br/

7. Programa das Nações Unidas para o Desenvolvimento do Brasil [homepage on the Internet]. Atlas do desenvolvimento humano no Brasil 2013 [accessed Mar 2 2014]. Available from: http://www.atlasbrasil.org.br/2013/pt/

8. Zambon MP, Antonio MARGM, Mendes RT, Barros Filho AA. Hipotireoidismo adquirido tratado como obesidade exógena: a importância do controle do crescimento. Rev Paul Pediatr. 2009;27(1):106-9. DOI: http://dx.doi.org/10.1590/S010305822009000100016

9. O'Brien SH, Holubkov R, Reis EC. Identification, evaluation, and management of obesity in an academic primary care center. Pediatrics. 2004;114(2):e154-9.

10. Pacifico L, Anania C, Ferraro F, Andreoli GM, Chiesa C. Thyroid function in childhood obesity and metabolic comorbidity. Clin Chim Acta. 2012;413(3-4):396-405. DOI: http://dx.doi.org/10.1016/j.cca.2011.11.013

11. Marwaha RK, Tandon N, Garg MK, Ganie MA, Narang A, Mehan N, et al. Impact of body mass index on thyroid functions in Indian children. Clin Endocrinol (Oxf). 2013;79(3):424-8. DOI: http://dx.doi.org/10.1111/cen.12148

12. Emokpae MA, Adeleke SI, Uwumarongie HO. Subclinical hypothyroidism in childhood obesity and its correlation with lipoproteins. Afr J Med Med Sci. 2011;40(4):361-5. PMID: 22783686

13. Brienza C, Grandone A, Di Salvo G, Corona AM, Di Sessa A, Pascotto C, et al. Subclinical hypothyroidism and myocardial function in obese children. Nutr Metab Cardiovasc Dis. 2013;23(9):898-902. DOI: http://dx.doi.org/10.1016/j. numecd.2012.04.006

14. Rotondi M, Magri F, Chiovato L. Thyroid and obesity: not a one-way interaction. J Clin Endocrinol Metab. 2011;96(2):344-6. DOI: http://dx.doi.org/10.1210/jc.2010-2515

15. Reinehr T. Obesity and thyroid function. Mol Cell Endocrinol. 2010;316(2):165-71. DOI: http://dx.doi.org/10.1016/j. mce.2009.06.005

16. Marras V, Casini MR, Pilia S, Carta D, Civolani P, Porcu M, et al. Thyroid function in obese children and adolescents. Horm Res Paediatr. 2010;73(3):193-7. DOI: http://dx.doi.org/10.1159/000284361

17. Reinehr T, Isa A, de Sousa G, Dieffenbach R, Andler W. Thyroid hormones and their relation to weight status. Horm Res. 2008;70(1):51-7. DOI: http://dx.doi.org/10.1159/000129678 
18. King K, O'Gorman C, Gallagher S. Thyroid dysfunction in children with Down syndrome: a literature review. Ir J Med Sci. 2014;183(1):1-6. DOI: http://dx.doi.org/10.1007/s11845-013-0994-y

19. Chen MH, Chen SJ, Su LY, Yang W. Thyroid dysfunction in patients with Down syndrome. Acta Paediatr Taiwan. 2007;48(4):191-5. ISSN: 1608-8115.

20. Bursell JDH, Warner JT. Interpretation of thyroid function in children. Paediatr Int Child Health. 2007;17(9):361-6. DOI: http://dx.doi.org/10.1016/j.paed.2007.06.010

21. Brenta G, Vaisman M, Sgarbi JA, Bergoglio LM, Andrada NC, Bravo PP, et al.; Task Force on Hypothyroidism of the Latin American Thyroid Society (LATS). Clinical practice guidelines for the management of hypothyroidism. Arq Bras Endocrinol Metabol. 2013;57(4):265-91. DOI: http://dx.doi.org/10.1590/S0004-27302013000400003

22. Chacko E, Graber E, Regelmann MO, Wallach E, Costin G, Rapaport R. Update on Turner and Noonan syndromes. Endocrinol Metab Clin North Am. 2012;41(4):713-34. DOI: http://dx.doi.org/10.1016/j.ecl.2012.08.007

23. Devernay M, Ecosse E, Coste J, Carel JC. Determinants of medical care for young women with Turner syndrome. J Clin Endocrinol Metab. 2009;94(9):3408-13. DOI: http://dx.doi.org/10.1210/jc.2009-0495

24. Dosi RV, Tandon N. A study on prevalence of thyroid auto-immunity in type 1 diabetes mellitus. J Indian Med Assoc. 2010;108(6):349-50.

25. Araujo J, Brandão LA, Guimarães RL, Santos S, Falcão EA, Milanese M, et al. Prevalence of autoimmune thyroid disease and thyroid dysfunction in young Brazilian patients with type 1 diabetes. Pediatr Diabetes. 2008;9(4 Pt 1):272-6. DOI: http:// dx.doi.org/10.1111/j.1399-5448.2008.00394.x

26. Kordonouri O, Maguire AM, Knip M, Schober E, Lorini R, Holl RW, et al. ISPAD Clinical Practice Consensus Guidelines 2009 Compendium. Other complications and associated conditions with diabetes in children and adolescents. Pediatr Diabetes 2009;10 Suppl. 12:204-10. DOI: http://dx.doi.org/10.1111/j.1399-5448.2009.00577.x

27. Silverstein J, Klingensmith G, Copeland K, Plotnick L, Kaufman F, Laffel L, et al.; American Diabetes Association. Care of children and adolescents with type 1 diabetes: a statement of the American Diabetes Association. Diabetes Care. 2005;28(1):186-212. DOI: http://dx.doi.org/10.2337/diacare.28.1.186

28. Kumar K, Kole AK, Karmakar PS, Ghosh A. The spectrum of thyroid disorders in systemic lupus erythematosus. Rheumatol Int. 2012;32(1):73-8. DOI: http://dx.doi.org/10.1007/s00296-010-1556-5

29. Harel L, Prais D, Uziel Y, Mukamel M, Hashkes P, Harel G, et al. Increased prevalence of antithyroid antibodies and subclinical hypothyroidism in children with juvenile idiopathic arthritis. J Rheumatol. 2006;33(1):164-6. ISSN: 0315-162X.

30. Stagi S, Giani T, Simonini G, Falcini F. Thyroid function, autoimmune thyroiditis and coeliac disease in juvenile idiopathic arthritis. Rheumatology (Oxford). 2005;44(4):517-20. DOI: http://dx.doi.org/10.1093/rheumatology/keh531

31. Pearce EN. Hypothyroidism and dyslipidemia: modern concepts and approaches. Curr Cardiol Rep. 2004;6(6):451-6. DOI: http://dx.doi.org/10.1007/s11886-004-0054-3

32. Catli G, Abaci A, Büyükgebiz A, Bober E. Subclinical hypothyroidism in childhood and adolescense. J Pediatr Endocrinol Metab. 2014;27(11-12):1049-57. DOI: http://dx.doi.org/10.1515/jpem-2014-0089

33. Tanis BC, Westendorp GJ, Smelt HM. Effect of thyroid substitution on hypercholesterolaemia in patients with subclinical hypothyroidism: a reanalysis of intervention studies. Clin Endocrinol (Oxf). 1996;44(6):643-9. DOI: http://dx.doi.org/10.1046/ j.1365-2265.1996.739560.x

34. Ladenson PW, Singer PA, Ain KB, Bagchi N, Bigos ST, Levy EG, et al. American Thyroid Association guidelines for detection of thyroid dysfunction. Arch Intern Med. 2000;160(11):1573-5. DOI: http://dx. doi.org/10.1001/archinte.160.11.1573

35. Garber JR, Cobin RH, Gharib H, Hennessey JV, Klein I, Mechanick JI, et al.; American Association of Clinical Endocrinologists and American Thyroid Association Taskforce on Hypothyroidism in Adults. Clinical practice guidelines for hypothyroidism in adults: cosponsored by the American Association of Clinical Endocrinologists and the American Thyroid Association. Endocr Pract. 2012;18(6):988-1028. DOI: http://dx.doi.org/10.4158/EP12280.GL

36. Gawlik A, Such K, Dejner A, Zachurzok A, Antosz A, Malecka-Tendera E. Subclinical hypothyroidism in children and adolescents: is it clinically relevant? Int J Endocrinol. 2015;2015:691071. DOI: http://dx.doi.org/10.1155/2015/691071 